
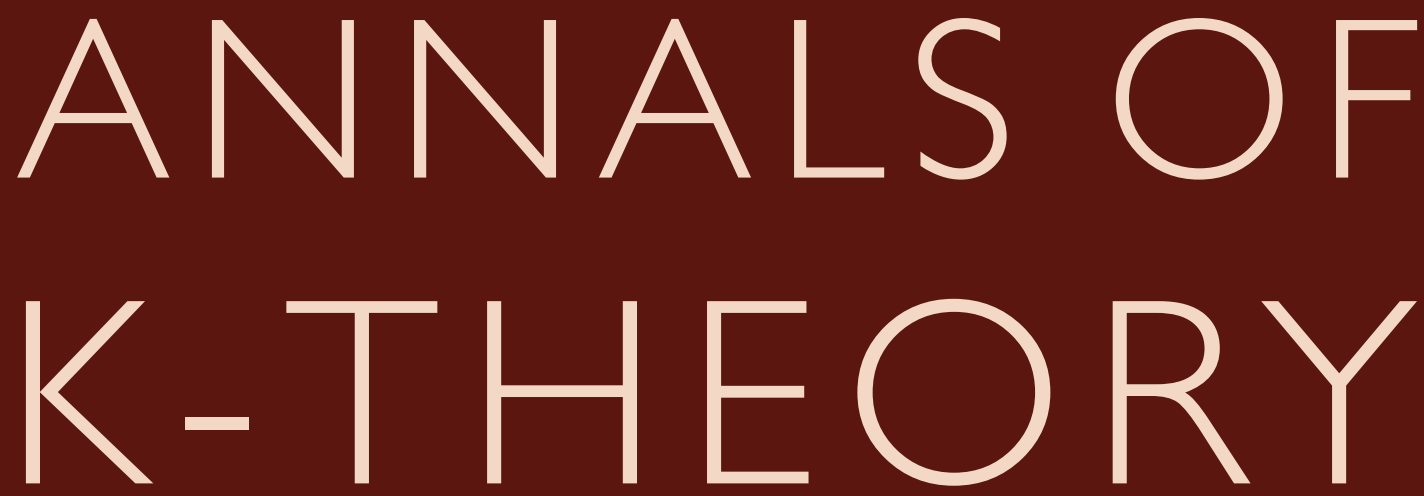

vol. 1 no. 42016

\title{
On the $K$-theory of linear groups
}

Daniel Kasprowski

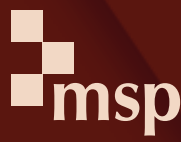

A JOURNAL OF THE K-THEORY FOUNDATION 


\title{
On the $K$-theory of linear groups
}

\author{
Daniel Kasprowski
}

\begin{abstract}
We prove that for a finitely generated linear group over a field of positive characteristic the family of quotients by finite subgroups has finite asymptotic dimension. We use this to show that the $K$-theoretic assembly map for the family of finite subgroups is split injective for every finitely generated linear group $G$ over a commutative ring with unit under the assumption that $G$ admits a finitedimensional model for the classifying space for the family of finite subgroups. Furthermore, we prove that this is the case if and only if an upper bound on the rank of the solvable subgroups of $G$ exists.
\end{abstract}

\section{Introduction}

For every group $G$ and every ring $A$ there is a functor $\mathbb{K}_{A}:$ Or $G \rightarrow \mathfrak{S p e c t r a}$ from the orbit category of $G$ to the category of spectra sending $G / H$ to (a spectrum weakly equivalent to) the $K$-theory spectrum $\mathbb{K}(A[H])$ for every subgroup $H \leq G$. For any such functor $F:$ Or $G \rightarrow \mathfrak{S p e c t r a , ~ a ~} G$-homology theory $\mathbb{F}$ can be constructed via

$$
\mathbb{F}(X):=\operatorname{Map}_{G}\left({ }_{-}, X_{+}\right) \wedge_{\mathrm{Or} G} F
$$

see [Davis and Lück 1998]. We will write $H_{n}^{G}(X ; F):=\pi_{n} \mathbb{F}(X)$ for its homotopy groups. The assembly map for the family of finite subgroups is the map

$$
H_{n}^{G}\left(\underline{E} G ; \mathbb{K}_{A}\right) \rightarrow H_{n}^{G}\left(\mathrm{pt} ; \mathbb{K}_{A}\right) \cong K_{n}(A[G])
$$

induced by the map $\underline{E} G \rightarrow$ pt. Here $\underline{E} G$ denotes the classifying space for the family of finite subgroups; see [Lück 2000]. The assembly map is a helpful tool for relating the $K$-theory of the group ring $A[G]$ to the $K$-theory of the group rings over the finite subgroups $H \leq G$. It can more generally be defined for any additive $G$-category instead of $A$; see [Bartels and Reich 2007]. Note that additive categories will always be small and that $K$-theory will always mean the nonconnective $K$-theory constructed by Pedersen and Weibel [1985].

MSC2010: 18F25, 19A31, 19B28, $19 \mathrm{G} 24$.

Keywords: $K$ - and $L$-theory of group rings, injectivity of the assembly map, linear groups. 
Theorem 1.1. Let $R$ be a commutative ring with unit and let $G \leq \mathrm{GL}_{n}(R)$ be finitely generated. If $G$ admits a finite-dimensional model for the classifying space $\underline{E} G$, then the assembly map

$$
H_{n}^{G}\left(\underline{E} G ; \mathbb{K}_{\mathcal{A}}\right) \rightarrow K_{n}(\mathcal{A}[G])
$$

is split injective for every additive $G$-category $\mathcal{A}$.

If $\mathcal{A}$ is an additive $G$-category with involution such that, for every virtually nilpotent subgroup $A \leq G$, there exists $i_{0} \in \mathbb{N}$ such that for $i \geq i_{0}$ we have $K_{-i}(\mathcal{A}[A])=0$, then the L-theoretic assembly map

$$
H_{n}^{G}\left(\underline{E} G ; \mathbb{L}_{\mathcal{A}}^{\langle-\infty\rangle}\right) \rightarrow L_{n}^{\langle-\infty\rangle}(\mathcal{A}[G])
$$

is split injective.

Theorem 1.1 implies the (generalized integral) Novikov conjecture for these groups by [Kasprowski 2015b, Section 6], since virtually nilpotent groups satisfy the Farrell-Jones conjecture by [Wegner 2015]. The (rational) Novikov conjecture for these groups is already known, by Guentner, Higson and Weinberger [Guentner et al. 2005], where it is shown that the Baum-Connes assembly map is split injective for linear groups.

We will use inheritance properties to reduce the proof of the theorem to the case where the ring $R$ has trivial nilradical and show that in this case the family $\{F \backslash G\}_{F \in \mathcal{F} \text { in }}$ has finite decomposition complexity, where $\mathcal{F}$ in denotes the family of finite subgroups of $G$. Then the theorem follows from the main theorem of [Kasprowski 2014]. For convenience, the necessary results of [Kasprowski 2014] are recalled in the Appendix.

By a result of Alperin and Shalen [1982], a finitely generated subgroup $G$ of $\mathrm{GL}_{n}(F)$, where $F$ is a field of characteristic zero, has finite virtual cohomological dimension if and only if there is a bound on the Hirsch rank of the unipotent subgroups of $G$. This in particular implies that it has a finite-dimensional model for the classifying space $\underline{E} G$. In positive characteristic, a finitely generated subgroup $G \leq \mathrm{GL}_{n}(F)$ always admits a finite-dimensional model for $\underline{E} G$, by [Degrijse and Petrosyan 2015, Corollary 5]. In Section 5 we prove the following generalization:

Proposition 1.2. Let $R$ be a commutative ring with unit and let $G \leq \mathrm{GL}_{n}(R)$ be finitely generated. Then $G$ admits a finite-dimensional model for $\underline{E} G$ if and only if there exists $N \in \mathbb{N}$ such that $l(A) \leq N$ for every solvable subgroup $A \leq G$, where $l(A)$ denotes the Hirsch rank of $A$.

Let $G$ be a solvable group and $1=G_{0} \unlhd G_{1} \unlhd \cdots G_{n-1} \unlhd G_{n}=G$ a normal series with abelian factors. The Hirsch rank (or Hirsch length) $l(G)$ of $G$ is

$$
l(G)=\sum_{i=1}^{n} \operatorname{dim}_{\mathbb{Q}} \mathbb{Q} \otimes_{\mathbb{Z}}\left(A_{i} / A_{i-1}\right) .
$$




\section{Finite decomposition complexity}

Let $X$ be a metric space. A decomposition $X=\bigcup_{i \in I} U_{i}$ is called $r$-disjoint, if $d\left(U_{i}, U_{j}\right)>r$ for all $i \neq j \in I$. We then denote the decomposition by

$$
X=\bigcup^{r \text {-disj. }} U_{i}
$$

A metric family is a set of metric spaces. A metric family $\left\{X_{i}\right\}_{i \in I}$ has finite asymptotic dimension uniformly if there exists an $n \in \mathbb{N}$ such that for every $r>0$ and $i \in I$ there exist decompositions

$$
X_{i}=\bigcup_{k=0}^{n} U_{i}^{k} \quad \text { and } \quad U_{i}^{k}=\bigcup_{j \in J_{i, k}}^{r \text {-disj. }} U_{i, j}^{k}
$$

such that $\sup _{i, j, k} U_{i, j}^{k}<\infty$.

Guentner, Tessera and Yu [Guentner et al. 2013] introduced the following generalization of finite asymptotic dimension:

Definition 2.1. Let $r>0$. A metric family $\mathcal{X}=\left\{X_{\alpha}\right\}_{\alpha \in A} r$-decomposes over a class of metric families $\mathfrak{D}$ if for every $\alpha \in A$ there exists a decomposition $X_{\alpha}=U_{\alpha}^{r} \cup V_{\alpha}^{r}$ and $r$-disjoint decompositions

$$
U_{\alpha}^{r}=\bigcup_{i \in I(r, \alpha)}^{r \text {-disj. }} U_{\alpha, i}^{r} \quad \text { and } \quad V_{\alpha}^{r}=\bigcup_{j \in J(r, \alpha)}^{r \text {-disj. }} V_{\alpha, j}^{r}
$$

such that the families $\left\{U_{\alpha, i}^{r}\right\}_{\alpha \in A, i \in I(r, \alpha)}$ and $\left\{V_{\alpha, j}^{r}\right\}_{\alpha \in A, j \in J(r, \alpha)}$ lie in $\mathfrak{D}$. A metric family $\mathcal{X}$ decomposes over $\mathfrak{D}$ if it $r$-decomposes over $\mathfrak{D}$ for all $r>0$.

Let $\mathfrak{B}$ denote the class of bounded families, i.e., $\mathcal{X} \in \mathfrak{B}$ if there exists $R>0$ such that diam $X<R$ for all $X \in \mathcal{X}$. We set $\mathfrak{D}_{0}=\mathfrak{B}$. For a successor ordinal $\gamma+1$ we define $\mathfrak{D}_{\gamma+1}$ to be the class of all metric families which decompose over $\mathfrak{D}_{\gamma}$. For a limit ordinal $\lambda$ we define

$$
\mathfrak{D}_{\lambda}=\bigcup_{\gamma<\lambda} \mathfrak{D}_{\gamma} .
$$

A metric family $\mathcal{X}$ has finite decomposition complexity $(F D C)$ if $\mathcal{X} \in \mathfrak{D}_{\gamma}$ for some ordinal $\gamma$.

A metric space $X$ has FDC if the family $\{X\}$ consisting only of $X$ has FDC. A group $G$ has FDC if it has FDC with any (and thus every) proper left-invariant metric.

A subfamily $\mathcal{Z}$ of a metric family $\mathcal{Y}$ is a metric family $\mathcal{Z}$ such that for each $Z \in \mathcal{Z}$ there exists $Y \in \mathcal{Z}$ with $Y \subseteq X$.

A map $F: \mathcal{X} \rightarrow \mathcal{Y}$ between metric families $\mathcal{X}$ and $\mathcal{Y}$ is a set of maps from elements of $\mathcal{X}$ to elements of $\mathcal{Y}$ such that every $X \in \mathcal{X}$ is the domain of at least 
one $f \in F$. The inverse image $F^{-1}(\mathcal{Z})$ of a subfamily $\mathcal{Z}$ of $\mathcal{Y}$ is the metric family $\left\{f^{-1}(Z) \mid Z \in \mathcal{Z}, f \in F\right\}$. A map $F: \mathcal{X} \rightarrow \mathcal{Y}$ is called uniformly expansive if there exists a nondecreasing function $\rho:[0, \infty) \rightarrow[0, \infty)$ such that for every $f: X \rightarrow Y$ in $F$ and every $x, y \in X$ we have

$$
d(f(x), f(y)) \leq \rho(d(x, y)) .
$$

We will use the following three results about FDC:

Theorem 2.2 [Guentner et al. 2013, Fibering theorem 3.1.4]. Let $\mathcal{X}$ and $\mathcal{Y}$ be metric families and let $F: \mathcal{X} \rightarrow \mathcal{Y}$ be uniformly expansive. Assume $\mathcal{Y}$ has FDC and that for every bounded subfamily $\mathcal{Z}$ of $\mathcal{Y}$ the inverse image $F^{-1}(\mathcal{Z})$ has $F D C$. Then $\mathcal{X}$ also has FDC.

Theorem 2.3 [Guentner et al. 2013, Theorem 4.1]. A metric space $X$ with finite asymptotic dimension has FDC.

While the above theorem is stated only for metric spaces it also holds for metric families which have finite asymptotic dimension uniformly.

Theorem 2.4 [Guentner et al. 2013, Theorem 3.1.7]. Let $X$ be a metric space, expressed as a union of finitely many subspaces $X=\bigcup_{i=0}^{n} X_{i}$. If the metric family $\left\{X_{i}\right\}_{i=0, \ldots, n}$ has $F D C$, so does $X$.

This theorem again holds for metric families instead of metric spaces, i.e., a metric family $\left\{\bigcup_{i=0}^{n} X_{i j}\right\}_{j \in J}$ has FDC if and only if the family $\left\{X_{i j}\right\}_{j \in J, i=0, \ldots, n}$ has FDC. We will also need the following two results about finite asymptotic dimension:

Lemma 2.5. Let $P: \mathcal{X} \rightarrow \mathcal{Y}$ be a family of maps such that for some $k>0$ each $p \in P$ is $k$-Lipschitz. Suppose that $\mathcal{Y}$ has finite asymptotic dimension uniformly and that for each $R>0$ the family

$$
\left\{p^{-1}\left(B_{R}(y)\right) \mid X \in \mathcal{X}, Y \in \mathcal{Y}, y \in Y,(p: X \rightarrow Y) \in P\right\}
$$

has finite asymptotic dimension uniformly. Then $\mathcal{X}$ has finite asymptotic dimension uniformly.

Lemma 2.6. Let $\mathcal{X}=\left\{U_{\alpha} \cup V_{\alpha}\right\}_{\alpha \in A}$ be a metric family. Then

$$
\operatorname{asdim} \mathcal{X}=\max \left\{\operatorname{asdim}\left\{U_{\alpha}\right\}_{\alpha}, \operatorname{asdim}\left\{V_{\alpha}\right\}_{\alpha \in A}\right\} .
$$

These results are [Roe 2003, Lemma 9.16 and Proposition 9.13], respectively, for metric families instead of metric spaces. The proofs are the same.

In the next section it will be more convenient to work with pseudometrics instead of metrics, i.e., allowing $d(x, y)=0$ for $x \neq y$. Finite asymptotic dimension and FDC are defined in the same way for pseudometrics. If $d$ is a pseudometric on $X$, then we can define a metric $d^{\prime}$ on $X$ by setting $d^{\prime}(x, y):=\max \{1, d(x, y)\}$ 
for $x \neq y$. The metric $d^{\prime}$ is proper (resp. left-invariant) if and only if $d$ is. It has finite asymptotic dimension (resp. FDC) if and only if $d$ does. Therefore, to show that a group has finite asymptotic dimension or FDC, it suffices to show this for $G$ equipped with a left-invariant proper pseudometric.

Notation 2.7. We write $\mathcal{F i n}_{G}$ for the set of finite subgroups of a group $G$. For a subgroup $H$ of $G$, by $\{F \backslash G\}_{F \in \mathcal{F} i n_{H}}$ we will mean the family of quotients of $G$ by all finite subgroups of $H$. When $H$ is the group of which we take the quotients, we will drop the subscript on $\mathcal{F}$ in, that is, $\{F \backslash G\}_{F \in \mathcal{F} \text { in }}=\{F \backslash G\}_{F \in \mathcal{F} i n_{G}}$.

\section{Linear groups over fields of positive characteristic}

In this section $K$ will always denote a field of positive characteristic. Every finitely generated subgroup $G$ of $\mathrm{GL}_{n}(K)$ has finite asymptotic dimension, by [Guentner et al. 2012, Theorem 3.1]. Here we want to show that the family $\{F \backslash G\}_{F \in \mathcal{F} \text { in }}$ has finite asymptotic dimension uniformly. We begin by recalling the argument from [Guentner et al. 2012].

A length function on a group $G$ is a function $l: G \rightarrow[0, \infty)$ such that, for all $g, h \in G$,

(1) $l(e)=0$,

(2) $l(g)=l\left(g^{-1}\right)$, and

(3) $l(g h) \leq l(g)+l(h)$.

We do not require that $l$ be proper, nor that $l(g)=0$ if and only if $g=e$. By setting $d(g, h):=l\left(g^{-1} h\right)$ we obtain a pseudometric.

A discrete norm on a field $K$ is a map $\gamma: K \rightarrow[0, \infty)$ satisfying that for all $x, y \in K$ we have

(1) $\gamma(x)=0$ if and only if $x=0$,

(2) $\gamma(x y)=\gamma(x) \gamma(y)$,

(3) $\gamma(x+y) \leq \max \{\gamma(x), \gamma(y)\}$,

and that the range of $\gamma$ on $K \backslash\{0\}$ is a discrete subgroup of the multiplicative group $(0, \infty)$.

Following [Guentner et al. 2005], we obtain for every discrete norm $\gamma$ on $K$ a length function $l_{\gamma}$ on $\mathrm{GL}_{n}(K)$ by

$$
l_{\gamma}(g)=\log \max _{i, j}\left\{\gamma\left(g_{i j}\right), \gamma\left(g^{i j}\right)\right\},
$$

where $g_{i j}$ and $g^{i j}$ are the matrix coefficients of $g$ and $g^{-1}$, respectively. By [Guentner et al. 2013, Propostion 5.2.4] the group $\mathrm{GL}_{n}(K)$ equipped with the pseudometric $d(g, h)=l_{\gamma}\left(g^{-1} h\right)$ has finite asymptotic dimension for every discrete norm $\gamma$. Let us review the proof. 
The subset $\mathcal{O}:=\{x \in K \mid \gamma(x) \leq 1\}$ is a subring of $K$ called the ring of integers and $\mathfrak{m}:=\{x \in K \mid \gamma(x)<1\}$ is a principal ideal in $\mathcal{O}$. Let $\pi$ be a fixed generator of $\mathfrak{m}$ and let $D$ denote the subgroup of diagonal matrices with powers of $\pi$ on the diagonal. Let $U$ denote the unipotent upper triangular matrices. By [Guentner et al. 2013, Lemma 5.2.5] the group $U$ has asymptotic dimension zero. We have $D \cong \mathbb{Z}^{n}$ and the restriction of $l_{\gamma}$ to $D$ is given by

$$
l_{\gamma}(a):=\max _{i}\left|k_{i}\right| \log \gamma\left(\pi^{-1}\right),
$$

where $a$ is the diagonal matrix with entries $\pi^{k_{i}}$ on the diagonal. The group $D$ therefore is quasi-isometric to $\mathbb{Z}^{n}$ with the standard metric and has asymptotic dimension $n$. The group $T:=D U$ is again a subgroup of $\operatorname{GL}_{n}(K)$ and $U \leq T$ is normal. Considering the extension $1 \rightarrow U \rightarrow T \rightarrow D \rightarrow 1$, we see that $T$ has finite asymptotic dimension.

Let $H$ be the subgroup of those $g \in \mathrm{GL}_{n}(F)$ for which the entries of $g$ and $g^{-1}$ are in $\mathcal{O}$. Then $\mathrm{GL}_{n}(K)=T H$ by [Guentner et al. 2005, Lemma 5]. For $h \in H$ let $h_{i j}$ and $h^{i j}$ denote the matrix coefficients of $h$ and $h^{-1}$, respectively. By definition, $\gamma\left(h_{i j}\right), \gamma\left(h^{i j}\right) \leq 1$ and thus

$$
0 \leq l_{\gamma}(h)=\log \max _{i j}\left\{\gamma\left(h_{i j}\right), \gamma\left(h^{i j}\right)\right\} \leq 0 .
$$

This implies that the inclusion $T \rightarrow \mathrm{GL}_{n}(K)$ is isometric and metrically onto, i.e., for every $g \in \mathrm{GL}_{n}(K)$ there exists a $t \in T$ with $d(g, t)=0$. Hence, $\mathrm{GL}_{n}(K)$ has finite asymptotic dimension with respect to the pseudometric $d$.

Lemma 3.1. For every discrete norm the family $\left\{F \backslash \mathrm{GL}_{n}(K)\right\}_{F}$, where $F$ ranges over all finite subgroups of $U$, has finite asymptotic dimension uniformly with respect to the associated pseudometric.

Proof. Let $F$ be a finite subgroup of $U$. Then we can consider the map

$$
F \backslash T \stackrel{\rho_{F}}{\longrightarrow} D .
$$

We want to apply Lemma 2.5 to the family $\left\{\rho_{F}: F \backslash T \stackrel{\rho}{\rightarrow} D\right\}_{F \in \mathcal{F} i n_{U}}$, For this we have to show that for every $R>0$ the family $\left\{\rho_{F}^{-1}\left(B_{R}(d)\right)\right\}_{d \in D, F \in \mathcal{F} i n_{U}}$ has finite asymptotic dimension uniformly. The preimage $\rho_{F}^{-1}(d)=\{F u d \mid u \in U\}$ of a point $d \in D$ is isometric to $(F)^{d} \backslash U$, by mapping $F u d$ to $d^{-1} F d d^{-1} u d$, where $(F)^{d}:=\left\{d^{-1} f d \mid f \in F\right\}$. Therefore, the preimage of $B_{R}(d)$ for any $R>0$ is a finite union of spaces isometric to spaces of the form $(F)^{d^{\prime}} \backslash U$ with $d^{\prime} \in D$. The number of spaces appearing in this union only depends on $R$ and not on $d$ (or $F$ ). Thus, by Lemma 2.6,

$$
\operatorname{asdim}\left\{\rho_{F}^{-1}\left(B_{R}(d)\right)\right\}_{d \in D, F \in \mathcal{F} i n_{U}}=\operatorname{asdim}\{F \backslash U\}_{F \in \mathcal{F} i n} .
$$


Since the inclusion $F \backslash T \rightarrow F \backslash \mathrm{GL}_{n}(K)$ is isometric and metrically onto, to prove the lemma it remains to show that the family $\{F \backslash U\}_{F \in \mathcal{F} \text { in }}$ has asymptotic dimension zero uniformly.

Let $R>0$ be given and let $\mathcal{S}$ denote the partition of $U$ into $r$-connected components, i.e., two elements $u, u^{\prime} \in U$ lie inside the same $S \in \mathcal{S}$ if and only if there exists a sequence $u_{0}, \ldots, u_{n}$ with $u=u_{0}, u^{\prime}=u_{n}$ and $d\left(u_{i-1}, u_{i}\right) \leq R$ for all $i=1, \ldots, n$. Since $U$ has asymptotic dimension zero we have that $r:=\sup _{S \in \mathcal{S}} \operatorname{diam} S<\infty$. Since the left action of $F$ on $U$ is isometric, if $f u=u^{\prime}$ for some $f \in F$ and $u, u^{\prime} \in U$, then $f$ maps the $r$-connected component of $u$ bijectively onto the $r$-connected component of $u^{\prime}$. This implies that every $r$-connected component of $F \backslash U$ is a quotient of an $r$-connected component of $U$ and in particular has diameter at most $r$. Therefore, the family $\{F \backslash U\}_{F \in \mathcal{F} \text { in }}$ has asymptotic dimension zero uniformly, as claimed.

Proposition 3.2. Let $G \leq \mathrm{GL}_{n}(K)$ be a finitely generated subgroup. Then for every discrete norm $\gamma$ the family $\{F \backslash G\}_{F \in \mathcal{F} \text { in }}$ has finite asymptotic dimension uniformly with respect to the associated pseudometric.

Proof. By the main theorem of [Alperin 1987] there exists a normal subgroup $G^{\prime} \leq G$ with index $\left[G: G^{\prime}\right]=: N<\infty$ such that every finite subgroup of $G^{\prime}$ is unipotent. Therefore, every finite subgroup $F \leq G^{\prime}$ is conjugate in $\mathrm{GL}_{n}(K)$ to a finite subgroup $F^{\prime} \leq U$. Let $g=t h$ with $t \in T, h \in H$ be such that $g^{-1} F^{\prime} g=F$. Since $U$ is normal in $T$, we have that $t^{-1} F^{\prime} t \leq U$ and we can assume $g \in H$ and in particular $l_{\gamma}(g)=0$. This implies that conjugation by $g$ is an isometry and induces an isometry between $F^{\prime} \backslash \mathrm{GL}_{n}(K)$ and $F \backslash \mathrm{GL}_{n}(K)$. By Lemma 3.1 the family $\left\{F^{\prime} \backslash \mathrm{GL}_{n}(K)\right\}_{F^{\prime} \in \mathcal{F} i n_{U}}$ has finite asymptotic dimension uniformly and, by the above isometry, the family $\left\{F \backslash \mathrm{GL}_{n}(K)\right\}_{F \in \mathcal{F}_{i} n_{G^{\prime}}}$ therefore also has finite asymptotic dimension uniformly. This also holds for the subfamily $\{F \backslash G\}_{F \in \mathcal{F} i n_{G^{\prime}}}$. Since $\left[G: G^{\prime}\right]=N$, every finite subgroup $\tilde{F}$ of $G$ has a normal subgroup $F$ of index at most $N$ lying in $G^{\prime}$. The quotient group $F \backslash \tilde{F}$ acts isometrically on $F \backslash G$. Thus, projecting the covers that give finite asymptotic dimension for $\{F \backslash G\}_{F \in \mathcal{F} i n_{G^{\prime}}}$ down to the quotient $\{\tilde{F} \backslash G\}_{\tilde{F} \in \mathcal{F} \text { in }}$ shows that this family still has finite asymptotic dimension uniformly.

Theorem 3.3. Let $G \leq \mathrm{GL}_{n}(K)$ be a finitely generated subgroup. There exists a proper, left-invariant metric on $G$ such that the family $\{F \backslash G\}_{F \in \mathcal{F} \text { in }}$ has finite asymptotic dimension uniformly.

Proof. The subring of $K$ generated by the matrix entries of a finite generating set for $G$ is a finitely generated domain $A$ with $G \leq \mathrm{GL}_{n}(A)$ and we may replace $K$ by the (finitely generated) fraction field of $A$; thus, we can assume that $K$ is a finitely generated field of positive characteristic. By [Guentner et al. 2012, Proposition 3.4], 
for every finitely generated subring $A$ of $K$ there exists a finite set $N_{A}$ of discrete norms such that for every $k \in \mathbb{N}$ the set

$$
B_{A}(k)=\left\{a \in A \mid \gamma(a) \leq e^{k} \text { for all } \gamma \in N_{A}\right\}
$$

is finite. Let $A$ again be the subring generated by the matrix entries of a finite generating set for $G$ and $N_{A}=\left\{\gamma_{1}, \ldots, \gamma_{q}\right\}$ be the finite set of discrete norms, as above. Consider the length function $l:=l_{\gamma_{1}}+\cdots+l_{\gamma_{q}}$. The pseudometric on $G$ defined by $d\left(g, g^{\prime}\right):=l\left(g^{-1} g^{\prime}\right)$ now is proper and left-invariant, and the diagonal embedding

$$
(G, d) \rightarrow\left(\mathrm{GL}_{n}(K), d_{\gamma_{1}}\right) \times \cdots \times\left(\mathrm{GL}_{n}(K), d_{\gamma_{q}}\right)
$$

is isometric when the product is given the sum metric. It suffices to show that the family

$$
\left\{F \backslash\left(\left(G, d_{\gamma_{1}}\right) \times \cdots \times\left(G, d_{\gamma_{q}}\right)\right)\right\}_{F \in \mathcal{F} \text { in }_{G}}
$$

has finite asymptotic dimension uniformly. Now let $F \leq G$ be finite and consider the projection

$$
F \backslash(G \times \cdots \times G) \stackrel{p}{\rightarrow} F \backslash G \times \cdots \times F \backslash G
$$

using the same metrics as above. The image has finite asymptotic dimension uniformly in $F$ by Proposition 3.2, and using Lemma 2.5 it suffices to show that the preimage of $B_{R}\left(F g_{1}\right) \times \cdots \times B_{R}\left(F g_{n}\right)$ under $p$ has finite asymptotic dimension uniformly. The preimage is a finite union of metric spaces of the form $F \backslash\left(F g_{1}^{\prime} \times F g_{n}^{\prime}\right)$ and the number of the spaces appearing in the union only depends on $R$, not on $F$ or $g_{1}, \ldots, g_{n}$. By the main theorem of [Alperin 1987] there exists a normal subgroup $G^{\prime} \unlhd \mathrm{GL}_{n}(A)$ with index $\left[\mathrm{GL}_{n}(A): G^{\prime}\right]=: N<\infty$ such that every finite subgroup of $G^{\prime}$ is unipotent. In particular, we have a normal unipotent subgroup $F^{\prime}:=G^{\prime} \cap F$ of $F$ of index at most $N$. The space $F g_{1}^{\prime} \times \cdots \times F g_{n}^{\prime}$ is a union of at most $N$ subspaces isometric to $F^{\prime} g_{1}^{\prime} \times \cdots \times F^{\prime} g_{n}^{\prime}$, and as in the proof of Proposition 3.2 there exists an isometry of these to $F_{1} \times \cdots \times F_{n}$ with $F_{i} \leq U$. By Lemma 2.6 this shows that $F g_{1}^{\prime} \times \cdots \times F g_{n}^{\prime}$ has asymptotic dimension zero uniformly in $F$. As in the proof of Lemma 3.1, we see that $F \backslash\left(F g_{1}^{\prime} \times \cdots \times F g_{n}^{\prime}\right)$ also has asymptotic dimension zero. This completes the proof of Theorem 3.3.

Remark 3.4. Note that the family $\{F \backslash G\}_{F \in \mathcal{F} \text { in }}$ has finite asymptotic dimension uniformly for some proper, left-invariant (pseudo)metric on $G$ if and only if it has finite asymptotic dimension for every proper, left-invariant metric on $G$.

\section{Linear groups over commutative rings with unit}

Lemma 4.1. Let $H_{1}$ and $H_{2}$ be groups such that $\left\{F \backslash H_{i}\right\}_{F \in \mathcal{F} \text { in }}$ has $F D C$ for $i=1,2$. Then $\left\{F \backslash\left(H_{1} \times H_{2}\right)\right\}_{F \in \mathcal{F} \text { in }}$ has $F D C$. 
Proof. Let proper, left-invariant metrics $d_{i}$ on $H_{i}$ be given and consider $H_{1} \times H_{2}$ with the metric $d_{1}+d_{2}$. Let $p_{i}: H_{1} \times H_{2} \rightarrow H_{i}$ denote the projection. Consider the uniformly expansive map

$$
\left\{F \backslash\left(H_{1} \times H_{2}\right)\right\}_{F \in \mathcal{F} \text { in }} \rightarrow\left\{\left(p_{1}(F) \times p_{2}(F)\right) \backslash\left(H_{1} \times H_{2}\right)\right\}_{F \in \mathcal{F} \text { in }} .
$$

Then the range has FDC by assumption and by the fibering theorem [Guentner et al. 2013, Theorem 3.1.4] it suffices to show that the family

$$
\left\{F \backslash\left(p_{1}(F) \times p_{2}(F)\right)\left(B_{R}\left(h_{1}\right) \times B_{R}\left(h_{2}\right)\right)\right\}_{h_{i} \in H_{i}, F \in \mathcal{F} i_{H_{1} \times H_{2}}}
$$

has FDC for every $R>0$. Every space in this family is a union of $\left|B_{R}\left(h_{1}\right) \times B_{R}\left(h_{2}\right)\right|$ many spaces of the form $F \backslash\left(p_{1}(F) \times p_{2}(F)\right)\left(h, h^{\prime}\right)$. The number $\left|B_{R}\left(h_{1}\right) \times B_{R}\left(h_{2}\right)\right|$ only depends on $R$, not on $h_{1}$ and $h_{2}$, and every space $F \backslash\left(p_{1}(F) \times p_{2}(F)\right)\left(h, h^{\prime}\right)$ is isometric to $(F)^{\left(h, h^{\prime}\right)} \backslash\left(p_{1}(F) \times p_{2}(F)\right)^{\left(h, h^{\prime}\right)}$, where $(F)^{\left(h, h^{\prime}\right)}$ is $\left(h, h^{\prime}\right)^{-1} F\left(h, h^{\prime}\right)$ and similarly for $\left(p_{1}(F) \times p_{2}(F)\right)^{\left(h, h^{\prime}\right)}$. By Theorem 2.4 it suffices to show that the family $\left\{F \backslash F^{\prime}\right\}_{F \leq F^{\prime}}$ has FDC, where $F \leq F^{\prime}$ ranges over all pairs of finite subgroups of $H_{1} \times H_{2}$. Let $\mathcal{S}_{R}$ denote the family of finite subgroups of $H_{1} \times H_{2}$ generated by elements from $B_{R}(e)$ and let $s_{R}:=\sup _{S \in \mathcal{S}_{R}} \operatorname{diam} S$. Let $F \leq H_{1} \times H_{2}$ be finite. Then for every $R>0$ the group $F$ is the $r$-disjoint union of the cosets of $\left\langle F \cap B_{R}(e)\right\rangle$ and each of these has diameter at most $s_{R}$. We see that the family of finite subgroups of $H_{1} \times H_{2}$ has asymptotic dimension zero uniformly. This implies that the above family $\left\{F \backslash F^{\prime}\right\}_{F \leq F^{\prime}}$ also has asymptotic dimension zero uniformly, since every $r$ connected component of $F \backslash F^{\prime}$ is a quotient of an $r$-connected component of $F^{\prime}$ and thus has uniformly bounded diameter.

Lemma 4.2 [Guentner et al. 2013, Lemma 5.2.3]. Let $R$ be a finitely generated commutative ring with unit and let $\mathfrak{n}$ be the nilpotent radical of $R$,

$$
\mathfrak{n}=\left\{r \in R \mid r^{n}=0 \text { for some } n\right\} .
$$

The quotient ring $S=R / \mathfrak{n}$ contains a finite number of prime ideals $\mathfrak{p}_{1}, \ldots, \mathfrak{p}_{k}$ such that the diagonal map

$$
S \rightarrow S / \mathfrak{p}_{1} \oplus \cdots \oplus S / \mathfrak{p}_{k}
$$

embeds $S$ into a finite direct sum of domains.

Theorem 4.3. Let $R$ be a commutative ring with unit and trivial nilradical and let $G$ be a finitely generated subgroup of $\operatorname{GL}(n, R)$. Then $\{F \backslash G\}_{F \in \mathcal{F} \text { in }}$ has $F D C$.

Proof. Because $G$ is finitely generated we can assume that $R$ is finitely generated as well. Since the nilradical of $R$ is trivial, we have $R=S$ in the notation of the previous lemma and there is an embedding

$\mathrm{GL}_{n}(S) \hookrightarrow \mathrm{GL}_{n}\left(S / \mathfrak{p}_{1}\right) \times \cdots \times \mathrm{GL}_{n}\left(S / \mathfrak{p}_{k}\right) \hookrightarrow \mathrm{GL}_{n}\left(Q\left(S / \mathfrak{p}_{1}\right)\right) \times \cdots \times \mathrm{GL}_{n}\left(Q\left(S / \mathfrak{p}_{k}\right)\right)$, 
where $Q\left(S / \mathfrak{p}_{i}\right)$ is the quotient field of $S / \mathfrak{p}_{i}$. Let $G_{i}$ be the image of the group $G$ in $\mathrm{GL}_{n}\left(Q\left(S / \mathfrak{p}_{i}\right)\right)$. If $S / \mathfrak{p}_{i}$ has positive characteristic, the family $\left\{F \backslash G_{i}\right\}_{F \in \mathcal{F} \text { in }}$ has FDC by Theorem 3.3. If $S / \mathfrak{p}_{i}$ has characteristic zero, then $G_{i}$ is virtually torsion-free by Selberg's lemma and thus $\left\{F \backslash G_{i}\right\}_{F \in \mathcal{F} \text { in }}$ has FDC by [Kasprowski 2015a, Theorem 4.10]. Now Lemma 4.1 implies that the family $\{F \backslash G\}_{F \in \mathcal{F} \text { in }}$ also has FDC.

Proof of Theorem 1.1. This follows directly from Theorem 4.3 and [Kasprowski 2014, Theorems 3.2.2 and 3.3.1] if $R$ has trivial nilradical. Note that these theorems are stronger than the similar [Kasprowski 2015a, Theorems A and 9.1], where an upper bound on the order of the finite subgroups is needed. For convenience we show in the Appendix how the results from [Kasprowski 2015a] can be used to prove the theorems from [Kasprowski 2014].

If the nilradical $\mathfrak{n}$ of $R$ is nontrivial, we have an exact sequence

$$
1 \rightarrow\left(1+M_{n}(\mathfrak{n})\right) \cap G \rightarrow G \rightarrow H \rightarrow 1,
$$

where $H$ denotes the image of $G$ in $\mathrm{GL}_{n}(R / \mathfrak{n})$. Now the $K$-theoretic assembly map for $H$ is split injective and $\left(1+M_{n}(\mathfrak{n})\right) \cap G$ is nilpotent. Therefore, the preimage of every virtually cyclic subgroup of $H$ is virtually solvable and satisfies the Farrell-Jones conjecture, by [Wegner 2015]. By [Kasprowski 2015b, Proposition 4.1] this implies that the $K$-theoretic assembly map for $G$ is split injective as well. The $L$-theory version of the theorem follows in the same way from the results in [Kasprowski 2015b, Section 6].

\section{Dimension of the classifying space}

In this section we want to prove Proposition 1.2. We will need the following result about classifying spaces. The proof is the same as the proof of [Lück 2000, Theorem 3.1].

Theorem 5.1. Let $1 \rightarrow K \rightarrow G \stackrel{\pi}{\rightarrow} Q \rightarrow 1$ be an exact sequence of groups. Assume that $Q$ admits a finite-dimensional model for $\underline{E} Q$ and that there exists $N \in \mathbb{N}$ such that for every finite subgroup $F \in Q$ the preimage admits a model for $\underline{E} \pi^{-1}(F)$ of dimension at most $N$. Then there exists a finite-dimensional model for $\underline{E} G$.

Proof of Proposition 1.2. For a group $G$ let $\underline{c d} G$ be the shortest length of a projective resolution of $\mathbb{Z}$ as a $\mathbb{Z}[G]$-module and let $\underline{h d} G$ be the shortest length of a flat resolution of $\mathbb{Z}$ of $\mathbb{Z}$ as a $\mathbb{Z}[G]$-module. Let $g d G$ denote the minimal dimension of a model for $\underline{E} G$. For a countable group $G \overline{\text { by }}$ [Nucinkis 2004, Theorem 4.1] we have

Furthermore,

$$
\underline{h d} G \leq \underline{c d} G \leq \underline{h d} G+1
$$

$$
\underline{c d} G \leq \underline{g d} G \leq \max \{\underline{c d} G, 3\},
$$


where the first inequality follows from taking the cellular chain complex of $\underline{E} G$ as a resolution and the second inequality follows from [Lück 1989, Theorem 13.19]. By [Flores and Nucinkis 2007, Theorem 1], for a solvable group with finite Hirsch length $l(G)$ it holds that $l(G)=\underline{h d} G$. Note that Flores and Nucinkis use Hillman's definition of the Hirsch rank for an elementary amenable group. It can be shown by a simple transfinite induction that for solvable groups this agrees with the definition given in the introduction. Furthermore, every solvable group with infinite Hirsch length has a subgroup with arbitrary large Hirsch length. In particular, the existence of a finite-dimensional model $X$ for $\underline{E} G$ directly implies that the Hirsch rank of the solvable subgroups of $G$ is bounded by $\operatorname{dim} X$. It remains to prove the other direction.

Let $R$ be a fixed commutative ring with unit and let $G \leq \mathrm{GL}_{n}(R)$ be finitely generated with $N \in \mathbb{N}$ an upper bound on the Hirsch rank of the solvable subgroups of $G$. Since $G$ is finitely generated, we can assume that $R$ is also finitely generated and let $\mathfrak{n}, S$ and $\mathfrak{p}_{1}, \ldots, \mathfrak{p}_{k}$ be as in Lemma 4.2. Furthermore, let $H$ denote the image of $G$ in $\mathrm{GL}_{n}(S)$ and $p: \mathrm{GL}_{n}(R) \rightarrow \mathrm{GL}_{n}(S)$ the projection. Let $A$ be a finitely generated abelian subgroup of $H$. Then $p^{-1}(A)$ is solvable. This implies that the rank of the finitely generated abelian subgroups of $H$ is also bounded by $N$.

First let us show that $H$ admits a finite-dimensional model for $\underline{E} H$. By Lemma 4.2 $H$ embeds into $\mathrm{GL}_{n}\left(S / \mathfrak{p}_{1}\right) \times \cdots \times \mathrm{GL}_{n}\left(S / \mathfrak{p}_{k}\right)$ and, since $H$ is finitely generated, we can assume that all the domains $S / \mathfrak{p}_{i}$ are as well. Order them in such a way that $S / \mathfrak{p}_{1}, \ldots, S / \mathfrak{p}_{q}$ are of positive characteristic and $S / \mathfrak{p}_{q+1}, \ldots, S / \mathfrak{p}_{k}$ are of characteristic zero. Then $\mathrm{GL}_{n}\left(S / \mathfrak{p}_{q+1}\right) \times \cdots \times \mathrm{GL}_{n}\left(S / \mathfrak{p}_{k}\right)$ embeds into $\mathrm{GL}_{n(k-q)}(\mathbb{C})$. Let $\pi$ denote the projection of $H$ to $\mathrm{GL}_{n}\left(S / \mathfrak{p}_{1}\right) \times \cdots \times \mathrm{GL}_{n}\left(S / \mathfrak{p}_{q}\right)$ and let $\pi_{i}$ denote the projection of $H$ to $\mathrm{GL}_{n}\left(S / \mathfrak{p}_{i}\right)$ for $i=1, \ldots, q$; then $\pi_{i}(H)$ admits a finite-dimensional model $E_{i}$ for $\underline{E} \pi_{i}(H)$, by [Degrijse and Petrosyan 2015, Corollary 5], and thus $E_{1} \times \cdots \times E_{q}$ is a finite-dimensional model for $\underline{E} \pi(H)$. By Theorem 5.1 it remains to show that for every finite subgroup $F \in \pi(H)$ the preimage $\pi^{-1}(F)$ admits a finite-dimensional model with dimension bounded uniformly in $F$. Let $\rho$ denote the projection from $H$ to $\mathrm{GL}_{n(k-q)}(\mathbb{C})$. Then $\rho(H)$ is virtually torsion-free, by Selberg's lemma [1960]. The group $\rho(\operatorname{ker} \pi)$ is isomorphic to ker $\pi$ and thus $N$ is a bound on the rank of its finitely generated abelian subgroups. Furthermore, $\rho(\operatorname{ker} \pi)$ has finite index in $\rho\left(\pi^{-1}(F)\right)$ for every finite subgroup $F \leq \pi(H)$. Thus, the rank of the finitely generated abelian subgroups of $\rho\left(\pi^{-1}(F)\right)$ is also bounded by $N$. By [Kasprowski 2015b, Proposition 3.1] this implies that the rank of the finitely generated unipotent subgroups of $\rho\left(\pi^{-1}(F)\right)$ is bounded by $\frac{1}{2} N(N+1)$. This implies that $\rho\left(\pi^{-1}(F)\right)$ has finite virtual cohomological dimension bounded uniformly in $F$; see [Alperin and Shalen 1982, Remark after Theorem 3.3]. The order of the finite subgroups in $\rho\left(\pi^{-1}(F)\right)$ is bounded uniformly in $F$ since they are all contained inside the virtually torsion- 
free group $\rho(H)$. By Theorem 1.10 of [Lück 2000] this implies that there exist finite-dimensional models for $\underline{E} \rho\left(\pi^{-1}(F)\right)$ with dimension bounded uniformly in $F$ and, since $\rho: \pi^{-1}(F) \rightarrow \rho\left(\pi^{-1}(F)\right)$ has finite kernel, they are also models for $E \pi^{-1}(F)$. This completes the proof that $H$ admits a finite-dimensional model for $\underline{E} H$.

For every finite subgroup $F \leq H$, its preimage $A$ in $G$ is virtually nilpotent and thus elementary amenable, and the Hirsch rank of $A$ is bounded by $N$. By the inequalities from the beginning of the proof this implies that there is a model for $\underline{E} A$ of dimension at most $N+2$. Using Theorem 5.1 again we conclude that there exists a finite-dimensional model for $\underline{E} G$.

\section{Appendix}

In this appendix we want to prove the following:

Theorem A.1 [Kasprowski 2014, Theorem 3.2.2]. Let $G$ be a discrete group such that $\{H \backslash G\}_{H \in \mathcal{F} \text { in }}$ has $F D C$ and let $\mathcal{A}$ be a small additive $G$-category. Assume that there is a finite-dimensional G-CW model for the classifying space for proper $G$-actions $\underline{E} G$. Then the assembly map in algebraic K-theory

is a split injection.

$$
H_{*}^{G}\left(\underline{E} G ; \mathbb{K}_{\mathcal{A}}\right) \rightarrow K_{*}(\mathcal{A}[G])
$$

The analogous result in $L$-theory [Kasprowski 2014, Theorem 3.3.1] follows in the same way from the results of [Kasprowski 2015a]. We will use the notation introduced in [Kasprowski 2015a]. Note that, in the appendix, metrics are allowed to take on the value $\infty$. We will need the following equivariant version of FDC.

Definition A.2. An equivariant metric family is a family $\left\{\left(X_{\alpha}, G_{\alpha}\right)\right\}_{\alpha \in A}$, where $G_{\alpha}$ is a group and $X_{\alpha}$ is a metric $G_{\alpha}$-space.

Definition A.3. An equivariant metric family $\mathcal{X}=\left\{\left(X_{\alpha}, G_{\alpha}\right)\right\}_{\alpha \in A}$ decomposes over a class of equivariant metric families $\mathfrak{D}$ if for every $r>0$ and every $\alpha \in A$ there exists a decomposition $X_{\alpha}=U_{\alpha}^{r} \cup V_{\alpha}^{r}$ into $G_{\alpha}$-invariant subspaces and $r$ disjoint decompositions

$$
U_{\alpha}^{r}=\bigcup_{i \in I(r, \alpha)}^{r \text {-disj. }} U_{\alpha, i}^{r} \quad \text { and } \quad V_{\alpha}^{r}=\bigcup_{j \in J(r, \alpha)}^{r \text {-disj. }} V_{\alpha, j}^{r}
$$

such that $G_{\alpha}$ acts on $I(r, \alpha)$ and $J(r, \alpha)$ and, for every $g \in G_{\alpha}$, we have $g U_{\alpha, i}^{r}=$ $U_{\alpha, g i}^{r}$ and $g V_{\alpha, j}^{r}=V_{\alpha, g j}^{r}$. Furthermore, the families

$$
\left\{\left(\coprod_{i \in I(r, \alpha)} U_{\alpha, i}^{r}, G_{\alpha}\right)\right\}_{\alpha \in A} \text { and }\left\{\left(\coprod_{j \in J(r, \alpha)} V_{\alpha, j}^{r}, G_{\alpha}\right)\right\}_{\alpha \in A}
$$

have to lie in $\mathfrak{D}$. 
Notice that the underlying sets of $U_{\alpha}^{r}$ and $\bigsqcup_{i \in I(r, \alpha)} U_{\alpha, i}^{r}$ are canonically isomorphic and in this sense the $G_{\alpha}$-action on $\bigsqcup_{i \in I(r, \alpha)} U_{\alpha, i}^{r}$ is the same as the action on $U_{\alpha}^{r}$, only the metric has changed.

Definition A.4. An equivariant metric family $\mathcal{X}$ is called semibounded if there exists $R>0$ such that for all $(X, G) \in \mathcal{X}$ and $x, y \in X$ we have $d(x, y)<R$ or $d(x, y)=\infty$.

Let $e \mathfrak{B}$ denote the class of semibounded equivariant families. We set $e \mathfrak{D}_{0}=e \mathfrak{B}$ and, given a successor ordinal $\gamma+1$, we define $e \mathfrak{D}_{\gamma+1}$ to be the class of all equivariant metric families which decompose over $e \mathcal{D}_{\gamma}$. For a limit ordinal $\lambda$ we define

$$
e \mathfrak{D}_{\lambda}=\bigcup_{\gamma<\lambda} e \mathfrak{D}_{\gamma} .
$$

An equivariant metric family $\mathcal{X}$ has finite decomposition complexity $(F D C)$ if $\mathcal{X}$ lies in $e \mathfrak{D}_{\gamma}$ for some ordinal $\gamma$.

Note that the equivariant metric family $\left\{\left(X_{\alpha},\{e\}\right)\right\}_{\alpha \in A}$ has FDC if and only if the metric family $\left\{X_{\alpha}\right\}_{\alpha \in A}$ has FDC.

A metric family $\left\{X_{\alpha}\right\}_{\alpha \in A}$ has uniformly bounded geometry if for every $R>0$ there exists $N \in \mathbb{N}$ such that, for every $\alpha \in A$ and $U \subseteq X_{\alpha}$ with $\operatorname{diam}(U) \leq R$, the set $U$ contains at most $N$ elements.

The following is a generalization of Ramras, Tessera and Yu [Ramras et al. 2014, Theorem 6.4]. The proof is analogous to the proof of theirs and can be found in [Kasprowski 2014]. The additive $G$-category $\mathcal{A}_{G}(X)$ is defined in [Kasprowski 2015a, Definition 5.1] and $\mathcal{A}_{G}^{G}(X)$ denotes the fixed-point category. For a definition of the bounded product see [Kasprowski 2015a, Definition 5.11].

Theorem A.5. Let $\mathcal{X}=\left\{\left(X_{\alpha}, G_{\alpha}\right)\right\}_{\alpha \in A}$ be an equivariant family with FDC, and let the family $\left\{X_{\alpha}\right\}_{\alpha \in A}$ have bounded geometry uniformly. Then

$$
\operatorname{colim}_{s} K_{n}\left(\prod_{\alpha \in A}^{b d} \mathcal{A}_{G_{\alpha}}^{G_{\alpha}}\left(P_{s} X_{\alpha}\right)\right)=0
$$

for all $n \in \mathbb{Z}$, where the colimit is taken over the maps induced by the inclusion of the respective Rips complexes $P_{s} X_{\alpha}$.

Furthermore, recall the following:

Theorem A.6 [Kasprowski 2015a, Theorem 7.6]. Let $G$ be a discrete group admitting a finite-dimensional model for $\underline{E} G$ and let $X$ be a simplicial $G-C W$ complex with a proper $G$-invariant metric. Assume that, for every $G$-set $J$ with finite stabilizers,

$$
\underset{K}{\operatorname{colim}} K_{n}\left(\prod_{j \in J}^{b d} \mathcal{A}_{G}(G K)\right)^{G}=0,
$$


where the colimit is taken over all finite subcomplexes $K \subseteq X$. Then the assembly map

$$
H_{*}^{G}\left(X ; \mathbb{K}_{\mathcal{A}}\right) \rightarrow K_{*}(\mathcal{A}[G])
$$

is a split injection.

Proposition A.7 [Kasprowski 2014, Proposition 3.2.1]. Let $G$ be a group such that the metric family $\{H \backslash G\}_{H \in \mathcal{F} \text { in }}$ has $F D C$. Then the equivariant metric family $\{(G, H)\}_{H \in \mathcal{F} \text { in }}$ has FDC as well.

Proof. Let $\left\{\left(X_{i}, G_{i}\right)\right\}_{i \in I}$ be an equivariant metric family with $G_{i} \leq G$ a finite subgroup and assume $X_{i} \subseteq \bigsqcup_{A_{i}} G$ is a $G_{i}$-invariant subspace, where $A_{i}$ is a $G_{i}$-set. We prove by induction on the decomposition complexity that the family $\left\{\left(X_{i}, G_{i}\right)\right\}_{i \in I}$ lies in $e \mathfrak{D}_{\gamma+1}$ if $\left\{G_{i} \backslash X_{i}\right\}_{i \in I} \in \mathfrak{D}_{\gamma}$. For the start of the induction let $\left\{G_{i} \backslash X_{i}\right\}_{i \in I}$ be in $\mathfrak{D}_{0}=\mathfrak{B}$. Since $G_{i} \backslash X_{i}$ is bounded, there is $a_{i} \in A_{i}$ with $X_{i} \subseteq \bigsqcup_{G_{i}} a_{i} G$. Then there exist $R>0$ and $Y_{i} \subseteq G=\bigsqcup_{\left\{a_{i}\right\}} G \subseteq \bigsqcup_{A_{i}} G$ with $\operatorname{diam} Y_{i}<R$ for all $i \in I$ such that $X_{i}=G_{i} Y_{i} \subseteq \bigsqcup_{A_{i}} G$. Let $G_{i}^{\prime} \subseteq G_{i}$ be the stabilizer of $a_{i}$. Then

$$
X_{i} \cong \coprod_{[g] \in G_{i} / G_{i}^{\prime}} g G_{i}^{\prime} Y_{i} \quad \text { with } \quad G_{i}^{\prime} Y_{i} \subseteq G
$$

Let $r>0$ be given and define $\mathcal{S}_{r}:=\left\{H \in \mathcal{F}\right.$ in $\left.\mid H=\langle S\rangle, S \subseteq B_{2 R+r}(e)\right\}$ and $k:=\max _{H \in \mathcal{S}}|H|$. Let $g_{i} \in Y_{i}$ be a fixed base point. Let $H_{i} \leq G_{i}^{\prime}$ be the subgroup generated by those $g \in G_{i}^{\prime}$ with $d\left(Y_{i}, g Y_{i}\right)<r$. For these $g$ we have $d\left(e, g_{i}^{-1} g g_{i}\right)<2 R+r$. Therefore, $g_{i}^{-1} H_{i} g_{i} \in \mathcal{S}_{r}$ and $\left|H_{i}\right| \leq k$. We have the decomposition

$$
X_{i}=\bigcup_{[g] \in G_{i} / H_{i}} g H_{i} Y_{i}
$$

This decomposition is $r$-disjoint, since $d\left(g h y, g^{\prime} h^{\prime} y^{\prime}\right)<r$ with $g, g^{\prime} \in G_{i}, h, h^{\prime} \in H_{i}$ and $y, y^{\prime} \in Y_{i}$ implies that $d\left(Y_{i}, h^{-1} g^{-1} g^{\prime} h^{\prime} Y_{i}\right)<r$ and so, by definition, the element $h^{-1} g^{-1} g^{\prime} h^{\prime}$ lies in $H_{i}$, which is equivalent to $g H_{i}=g^{\prime} H_{i}$.

By definition of $H_{i}$ each $h \in H_{i}$ can be written as $h=g_{1} \cdots g_{l}$ with $l \leq|H| \leq k$ and $g_{j}$ such that $d\left(Y_{i}, g_{j} Y_{i}\right)<r$. For every $y, y^{\prime} \in Y_{i}$, by left-invariance and the triangle inequality we obtain

$$
\begin{aligned}
d\left(y, h y^{\prime}\right) & \leq d\left(y, g_{1} y^{\prime}\right)+d\left(g_{1} y^{\prime}, g_{1} g_{2} y^{\prime}\right)+\cdots+d\left(g_{1} \cdots g_{l-1} y^{\prime}, h y^{\prime}\right) \\
& =d\left(y, g_{1} y^{\prime}\right)+d\left(y^{\prime}, g_{2} y^{\prime}\right)+\cdots+d\left(y^{\prime}, g_{l} y^{\prime}\right)<l r .
\end{aligned}
$$

Therefore diam $g H_{i} Y_{i}=\operatorname{diam} H_{i} Y_{i}<k r$. Thus, $\left\{\left(X_{i}, G_{i}\right)\right\}_{i \in I}$ is $r$-decomposable over $e \mathfrak{D}_{0}=e \mathfrak{B}$ for every $r>0$ and lies in $e \mathfrak{D}_{1}$.

If $\left\{G_{i} \backslash X_{i}\right\}_{i \in I}$ lies in $\mathfrak{D}_{\gamma+1}$, then it decomposes over $\mathfrak{D}_{\gamma}$ and the preimages under the projection $X_{i} \rightarrow G_{i} \backslash X_{i}$ give a decomposition of $\left\{\left(X_{i}, G_{i}\right)\right\}$ over $e \mathfrak{D}_{\gamma+1}$ by the induction hypothesis. Here $G_{i}$ acts trivially on the index set of the decomposition. The induction step for limit ordinals is trivial. 
Proof of Theorem A.1. By [Kasprowski 2015a, Proposition 1.5], $G$ admits a finitedimensional model $X$ for $\underline{E} G$ with a left-invariant proper metric. By Theorem A.6 we have to show that

$$
\underset{K}{\operatorname{colim}} K_{n}\left(\prod_{j \in J}^{b d} \mathcal{A}_{G}(G K)\right)^{G}=0,
$$

where the colimit is taken over all finite subcomplexes $K \subseteq X$. Since the category $\left(\prod_{j \in J}^{b d} \mathcal{A}_{G}(G K)\right)^{G}$ is equivalent to $\prod_{G j \in G \backslash J}^{b d} \mathcal{A}_{G}^{G_{j}}\left(P_{s} \bar{G}\right)$, where $G_{j}$ is the stabilizer of $j \in J$, this is equivalent to showing that, for every family of finite subgroups $\left\{G_{i}\right\}_{i \in I}$ over some index set $I$,

$$
\underset{K}{\operatorname{colim}} K_{n}\left(\prod_{i \in I}^{b d} \mathcal{A}_{G}^{G_{i}}(G K)\right)=0 .
$$

By [Kasprowski 2015a, Lemma 1.8 and Proposition 6.3], for every finite subcomplex $K \subseteq X$ there exists $K^{\prime} \subseteq X$ finite and $s>0$ with maps $G K \rightarrow P_{s}(G) \rightarrow G K^{\prime}$ such that the composition is metrically homotopic to the identity. In particular, the composition induces the identity in the $K$-theory of the associated controlled categories. Thus it remains to show

$$
\underset{s}{\operatorname{colim}} K_{n}\left(\prod_{i \in I}^{b d} \mathcal{A}_{G}^{G_{i}}\left(P_{S} G\right)\right)=0 .
$$

Since $\{(G, H)\}_{H \in \mathcal{F} \text { in }}$ has FDC by Proposition A.7 and the category $\mathcal{A}_{G}^{G_{i}}\left(P_{s} G\right)$ is equivalent to $\mathcal{A}_{G_{i}}^{G_{i}}\left(P_{s} G\right)$, this follows from Theorem A.5.

\section{Acknowledgments}

I would like to thank Christoph Winges and the referee for useful comments and suggestions. This work was supported by the Max Planck Society.

\section{References}

[Alperin 1987] R. C. Alperin, “An elementary account of Selberg's lemma”, Enseign. Math. (2) 33:3-4 (1987), 269-273. MR Zbl

[Alperin and Shalen 1982] R. C. Alperin and P. B. Shalen, "Linear groups of finite cohomological dimension", Invent. Math. 66:1 (1982), 89-98. MR Zbl

[Bartels and Reich 2007] A. Bartels and H. Reich, "Coefficients for the Farrell-Jones conjecture", Adv. Math. 209:1 (2007), 337-362. MR Zbl

[Davis and Lück 1998] J. F. Davis and W. Lück, "Spaces over a category and assembly maps in isomorphism conjectures in $K$ - and $L$-theory", $K$-Theory 15:3 (1998), 201-252. MR Zbl

[Degrijse and Petrosyan 2015] D. Degrijse and N. Petrosyan, "Bredon cohomological dimensions for groups acting on CAT(0)-spaces”, Groups Geom. Dyn. 9:4 (2015), 1231-1265. MR Zbl 
[Flores and Nucinkis 2007] R. J. Flores and B. E. A. Nucinkis, "On Bredon homology of elementary amenable groups”, Proc. Amer. Math. Soc. 135:1 (2007), 5-11. MR Zbl

[Guentner et al. 2005] E. Guentner, N. Higson, and S. Weinberger, "The Novikov conjecture for linear groups”, Publ. Math. Inst. Hautes Études Sci. 101 (2005), 243-268. MR Zbl

[Guentner et al. 2012] E. Guentner, R. Tessera, and G. Yu, "A notion of geometric complexity and its application to topological rigidity", Invent. Math. 189:2 (2012), 315-357. MR Zbl

[Guentner et al. 2013] E. Guentner, R. Tessera, and G. Yu, "Discrete groups with finite decomposition complexity", Groups Geom. Dyn. 7:2 (2013), 377-402. MR Zbl

[Kasprowski 2014] D. Kasprowski, On the K-theory of groups with finite decomposition complexity, Ph.D. thesis, Westfälische Wilhelms-Universtität, Münster, 2014, http://people.mpim-bonn.mpg.de/ kasprowski/ThesisKasprowski.pdf. Zbl

[Kasprowski 2015a] D. Kasprowski, "On the $K$-theory of groups with finite decomposition complexity", Proc. Lond. Math. Soc. (3) 110:3 (2015), 565-592. MR Zbl

[Kasprowski 2015b] D. Kasprowski, "On the $K$-theory of subgroups of virtually connected Lie groups”, Algebr. Geom. Topol. 15:6 (2015), 3467-3483. MR Zbl

[Lück 1989] W. Lück, Transformation groups and algebraic K-theory, Lecture Notes in Mathematics 1408, Springer, Berlin, 1989. MR Zbl

[Lück 2000] W. Lück, "The type of the classifying space for a family of subgroups", J. Pure Appl. Algebra 149:2 (2000), 177-203. MR Zbl

[Nucinkis 2004] B. E. A. Nucinkis, "On dimensions in Bredon homology", Homology Homotopy Appl. 6:1 (2004), 33-47. MR Zbl

[Pedersen and Weibel 1985] E. K. Pedersen and C. A. Weibel, "A nonconnective delooping of algebraic $K$-theory", pp. 166-181 in Algebraic and geometric topology (New Brunswick, NJ, 1983), edited by A. Ranicki et al., Lecture Notes in Mathematics 1126, Springer, Berlin, 1985. MR Zbl

[Ramras et al. 2014] D. A. Ramras, R. Tessera, and G. Yu, "Finite decomposition complexity and the integral Novikov conjecture for higher algebraic K-theory", J. Reine Angew. Math. 694 (2014), 129-178. MR Zbl

[Roe 2003] J. Roe, Lectures on coarse geometry, University Lecture Series 31, American Mathematical Society, Providence, RI, 2003. MR Zbl

[Selberg 1960] A. Selberg, "On discontinuous groups in higher-dimensional symmetric spaces", pp. 147-164 in Contributions to function theory (Bombay, 1960), edited by K. Chandrasekharan, Tata Institute of Fundamental Research, Bombay, 1960. MR Zbl

[Wegner 2015] C. Wegner, "The Farrell-Jones conjecture for virtually solvable groups", J. Topol. 8:4 (2015), 975-1016. MR Zbl

Received 8 May 2015. Revised 20 Sep 2015. Accepted 22 Oct 2015.

DANIEL KASPROWSKI: kasprowski@mpim-bonn.mpg.de

Max-Planck-Institut für Mathematik, Vivatsgasse 7, D-53111 Bonn, Germany 


\title{
ANNALS OF K-THEORY
}

\author{
msp.org/akt
}

EDITORIAL BOARD

\author{
Paul Balmer University of California, Los Angeles, USA \\ balmer@math.ucla.edu \\ Spencer Bloch University of Chicago, USA \\ bloch@math.uchicago.edu \\ Alain Connes Collège de France; Institut des Hautes Études Scientifiques; Ohio State University \\ alain@connes.org \\ Guillermo Cortiñas Universidad de Buenos Aires and CONICET, Argentina \\ gcorti@dm.uba.ar \\ Eric Friedlander University of Southern California, USA \\ ericmf@usc.edu \\ Max Karoubi Institut de Mathématiques de Jussieu - Paris Rive Gauche, France \\ max.karoubi@imj-prg.fr \\ Gennadi Kasparov Vanderbilt University, USA \\ gennadi.kasparov@vanderbilt.edu \\ Alexander Merkurjev \\ University of California, Los Angeles, USA \\ merkurev@math.ucla.edu \\ Amnon Neeman amnon.Australian National University \\ neeman@anu.edu.au \\ Jonathan Rosenberg (Managing Editor) \\ University of Maryland, USA \\ jmr@math.umd.edu \\ Marco Schlichting University of Warwick, UK \\ schlichting@warwick.ac.uk \\ Andrei Suslin Northwestern University, USA \\ suslin@math.northwestern.edu \\ Vladimir Voevodsky Institute for Advanced Studies, USA \\ vladimir@math.ias.edu \\ Charles Weibel (Managing Editor) \\ Rutgers University, USA \\ weibel@math.rutgers.edu \\ Guoliang Yu Texas A\&M University, USA \\ guoliangyu@math.tamu.edu \\ PRODUCTION \\ $\begin{array}{ll}\text { Silvio Levy } & \text { (Scientific Editor) } \\ \text { production@msp.org }\end{array}$
}

Annals of K-Theory is a journal of the K-Theory Foundation (ktheoryfoundation.org). The K-Theory Foundation acknowledges the precious support of Foundation Compositio Mathematica, whose help has been instrumental in the launch of the Annals of K-Theory.

See inside back cover or msp.org/ant for submission instructions.

The subscription price for 2016 is US $\$ 400 /$ year for the electronic version, and $\$ 450 /$ year (+\$25, if shipping outside the US) for print and electronic. Subscriptions, requests for back issues and changes of subscribers address should be sent to MSP.

Annals of K-Theory (ISSN 2379-1681 electronic, 2379-1683 printed) at Mathematical Sciences Publishers, 798 Evans Hall \#3840, c/o University of California, Berkeley, CA 94720-3840 is published continuously online. Periodical rate postage paid at Berkeley, CA 94704, and additional mailing offices.

AKT peer review and production are managed by EditFlow ${ }^{\circledR}$ from MSP.

PUBLISHED BY

mathematical sciences publishers nonprofit scientific publishing

http://msp.org/

(C) 2016 Mathematical Sciences Publishers 


\section{ANNALS \\ OF \\ K-THEORY}

2016

no. 4

Splitting the relative assembly map, Nil-terms and involutions

Wolfgang Lück and Wolfgang Steimle

Birational motives, I: pure birational motives

Bruno Kahn and Ramdorai Sujatha

On the $K$-theory of linear groups

Daniel Kasprowski

Standard norm varieties for Milnor symbols $\bmod p$

Dinh Huu Nguyen 